\title{
Interactive comment on "Quantifying the impacts of human water use and climate variations on recent drying of Lake Urmia basin: the value of different sets of spaceborne and in-situ data for calibrating a hydrological model" by Seyed-Mohammad Hosseini-Moghari et al.
}

\section{Anonymous Referee \#1}

Received and published: 11 October 2018

This manuscript uses WaterGAP Global Hydrology model to quantify the effects of human water use on inflow to Lake Urmia, lake water volume, and groundwater. The model was manually calibrated 4 for time using different observation data sets (remote sensing of irrigated area, monthly total water storage anomaly, insitu observations of stream flow, and groundwater levels from 284 wells). Strengths of the work include a focus on the pressing problem or Lake Urmia decline and identification of the effects on groundwater. With these strengths, there are also several issues that I feel need to 
be addressed to accept this manuscript for publication.

1. Is the finding that humans affected lake decline new? There have been several recent studies that report this finding (Alborzi et al. 2018; Chaudhari et al. 2018; Shadkam et al. 2016) and some of these studied used the same model inputs as this work and also report groundwater changes. What is new in this work?

2. Is a global hydrologic model appropriate for a basin level analysis? The description of how the model simulates relevant processes is scant. Given resonance times, how appropriate is the temporal spacing (daily) relevant to the spatial grid size? Is it computationally efficient to run a global model for 15 or 20 grid cells of interest? There needs to be a much stronger justification for why the modeling and calibration methods are the correct approaches to use to answer the motivating questions.

3. There is a lot of focus in the text on the multiple calibration variants run with different input data sets. What was learned from this activity? How do those results effect Lake Urmia management?

4. Also, what could one potentially learn from 4 model calibrations that use different calibration data and yield four different models?

5. What are the limitations of this study?

6. The discussion of uncertainty in the results needs to go much deeper and be more specific. This uncertainty is real and likely plays a large role in the interpretation of the results.

7. I found the writing difficult to follow in numerous places, particularly the results section. There are lots of acronyms, run-on sentences, and text that digresses from the section headers or topic sentences of paragraphs. The writing here made it difficult for me to see the main results and findings of the work.

Overall I recommend decline for publication.

Printer-friendly version

Discussion paper
Interactive

comment 
Additional line-by-line comments include: pp. 2-4. The first three figures recount results from prior work. I would much prefer to see figures and tables focus on new insights gained from the work. For example, new figures that show uncertainties.

p. 3, line 5. I think "somewhat recovered" is overstated. Hard to tell from Figure 3. Maybe stabilized.

p. 3, line 18. Is the value $-11.2 \mathrm{~mm} / \mathrm{yr}$ correct? It seems incredibly small. In The Hashemite Kingdom of Jordan, drawdowns are $1+\mathrm{m} / \mathrm{yr}$, in numerous wells. In the U.S., we talk about drawdowns of $\mathrm{ft} /$ year.

p. 6 , line 5. Only the anomalies? Or at all time periods? If the former, please explain what is meant by "anomaly", how determined, and why anomaly is the appropriate frame to discuss. I would want to calibrate a model across a range of conditions some of which might include anomalies.

p. 6, lines 18-28. I'm not familiar with WaterGAP. How does this model actually work? Explain.

p. 7. What is total water storage anomaly (TWSA)? This term seems rather central to the paper. Please explain.

p. 8, lines 13-18. This method of applying (1 - return flow multipliers) to the abstractions to estimate consumptive use assumes that water is used by only one water user. Is this a realistic assumption? If the return flow is used by another agricultural user and then again by a 3rd or 4th user, the basin-wide consumptive use fraction will be much different than the values reported. The large grid size magnifies this error. Table 1. How sensitive are study results to the values in this table?

p. 10. Lines 5-15. So the correction factors are needed because WaterGAP does not get the underlying physical hydrology correct? The correction is linear? Is the process causing the error also linear?

Printer-friendly version

p. 11, line 1. Which parameters were varied to calibrate this model?

Discussion paper 
p. 11 , line 4 . What is meant by optimal fit?

p. 15, line 2 and Table 3. Shouldn't these parameter values be the same across all the model variants? What is physically changing in the system that these parameter values would change across the model variants?

p. 16, lines 2-3. There could be a net groundwater abstraction but still areas where there is recharge. Is this an issue of coarse spatial resolution?

p. 18, lines 1-10. The discussion of uncertainty here is missing a fundamental point. Calibration can not help if the model structure is uncertain (or in error) or the temporal or spatial scaling of the model is mismatched to the modeled parameters of interest. This discussion also heads in a different direction than "what do we learn from the calibrations?" The text never explains what was learned. What was learned? Please discuss.

p. 18, lines 11-20. These statements are better placed in the introduction to justify the use of global hydrologic models. Still, why is a global model the appropriate choice when the domain of study is limited to one hydrologic basin (Urmia)?

p. 19 , line 22. What beta?

p. 19, line 30. "much less overestimated" means what?

p. 19, line 31-32. This doesn't make sense to me. How come it is ok to change the parameter in one model variant but not others?

p. 20, lines 1-5. I would expect to see better calibration with more observational data (i.e., streamflows and lake levels).

p. 20, line 22. I'm confused. The scenario "with reservoirs but without human water use" does not fit either of the two scenarios described in the prior sentence.

p. 20, line 24. What is meant by anomalies? This term has still not been defined. 
p. 21, line 17, "The lower lake water loss...." What are the loss terms besides evaporation? How are these other loss terms smaller when inflow is larger? Explain.

p. 21, lines 20-23. I don't follow this explanation. There are too many NAs in this sentence. What causes the difference between the naturalized and anthropogenic scenarios?

p. 21, lines 25-28. I don't follow. What is the connection between the first part of the sentence and the second part?

p. 21 , lines 28-32. Is a run-on sentence.

p. 21 , lines $32-21$. Put these ratios in context. What is desirable? Undesirable? What has implications for lake health? What values are acceptable?

p. 23 , line 15 . How can water storage be negative?

p. 23, line 18. This is an interesting result. It needs to be much more strongly emphasized. These cells are the locations where groundwater declines and there could be problems.

p. 23, lines 21-23. This qualification and limitation seems rather important. Why should the model results be trusted or used if the model does not get groundwater storage correct?

p. 23, lines 23-25. Run-on sentence. What is meant by the clause with maximum?

p. 24, lines 18-19. Is this result surprising? More calibration data means a better fit model. How does this result improve understanding of the Lake Urmia system?

p. 24, lines 25-28. Is this finding new? If so how? I feel the Urmia Lake Recovery Program has been working under the assumption that agricultural water use was a large contributor to lake decline and that they have been taking steps in recent years to address. 
p. 24 , line $29.90 \%$ of what?

p. 24, lines 19-24. I disagree. There are lots of other similar systems in the world - Great Salt Lake, Owens Lake, Dead Sea, Ural Sea, etc. each satisfy the first two criteria listed. What of these results is generalizable?

p. 24, lines 31-34. How do the model results inform the 2014-2017 trends? Also, how can climate change be constrained in this basin? Explain.

Figure 9. What is being shown in panels $A, B$, and $D$ ? The $y$-axis labels were mentioned in the text but never explained.

Figure A1a. The color scheme makes it difficult to differentiate grid cells. Use only three colors to differentiate the 3 types of storage. How can storage volume be negative? Data availability. I don't follow. If the authors do not have permission to share the data, then how can they share by author request? The HydroSat site underwritten by the University of Stuttgart is neat. What is the original source data for Urmia? Also, there is no water storage anomaly data for Urmia.

References Alborzi, A., Mirchi, A., Moftakhari, H., Mallakpour, I., Alian, S., Nazemi, A., Hassanzadeh, E., Mazdiyasni, O., Ashraf, S., Madani, K., Norouzi, H., Azarderakhsh, M., Mehran, A., Sadegh, M., Castelletti, A., and AghaKouchak, A. (2018). "Climate-informed environmental inflows to revive a drying lake facing meteorological and anthropogenic droughts." Environmental Research Letters, 13(8), 084010. http://stacks.iop.org/1748-9326/13/i=8/a=084010. Chaudhari, S., Felfelani, F., Shin, S., and Pokhrel, Y. (2018). "Climate and anthropogenic contributions to the desiccation of the second largest saline lake in the twentieth century." Journal of Hydrology, 560, 342-353. http://www.sciencedirect.com/science/article/pii/S0022169418302002. Shadkam, S., Ludwig, F., van Oel, P., Kirmit, Ç., and Kabat, P. (2016). "Impacts of climate change and water resources development on the declining inflow into Iran's Urmia Lake." Journal of Great Lakes Research, 42(5), 942-952. http://www.sciencedirect.com/science/article/pii/S0380133016301307. 
Interactive comment on Hydrol. Earth Syst. Sci. Discuss., https://doi.org/10.5194/hess-2018318, 2018.

\section{HESSD}

Interactive

comment 